\title{
On Binomial and Poisson Sums Arising from the Displacement of Randomly Placed Sensors
}

\author{
Michael Fuchs*, Louis Kao and Wan-Zhen Wu
}

\begin{abstract}
We re-visit the asymptotics of a binomial and a Poisson sum which arose as (average) displacement costs when moving randomly placed sensors to anchor positions. The first-order asymptotics of these sums were derived in several stages in a series of recent papers. In this paper, we give a unified approach based on the classical Laplace method with which one can also derive more terms in the asymptotic expansions. Moreover, in a special case, full asymptotic expansions can be given which even hold as identities. This will be proved by a combinatorial approach and systematic ways of computing all coefficients of these identities will be discussed as well.
\end{abstract}

\section{Introduction}

This paper is concerned with the asymptotics of the binomial sum

$$
S_{\operatorname{Bin}}(n):=\sum_{i=1}^{n} \int_{0}^{1}\left|x-t_{i}\right|^{a} i\left(\begin{array}{c}
n \\
i
\end{array}\right) x^{i-1}(1-x)^{n-i} \mathrm{~d} x,
$$

where $t_{i}:=i / n-1 /(2 n)$ and the Poisson sum

$$
S_{\text {Poi }}(n):=\sum_{i=0}^{n-2} \int_{0}^{\infty}\left|x-s_{i+1}\right|^{a} n e^{-n x} \frac{(n x)^{i}}{i !} \mathrm{d} x,
$$

where $s_{i}:=i / n$. In both sums, $a>0$ is an (arbitrary) real number.

The binomial sum was analyzed in a paper of Kapelko and Kranakis 6] and then studied in follow-up works of Kapelko [4,5]. The Poisson sum was investigated in a paper of Kapelko [3. See also Kranakis [8, Kranakis et al. [9] and Kranakis and Shaikhet 10,11 for background and related studies.

We will state the (final) results on the asymptotics of 1.1 and 1.2 from the papers of Kapelko below. However, before doing so, we will provide some background on why these sums have been considered.

Received October 30, 2019; Accepted May 21, 2020.

Communicated by Sen-Peng Eu.

2010 Mathematics Subject Classification. 05A16, 60C05, 68W40.

Key words and phrases. sensor, displacement cost, asymptotics, Laplace method, generating functions. All three authors have been partially supported by the grants MOST-104-2923-M-009-006-MY3 and MOST-107-2115-M-009-010-MY2.

*Corresponding author. 
Assume that $n$ sensors are randomly placed on the line at positions $X_{1}, \ldots, X_{n}$. In the binomial case, the $X_{i}$ are independent random variables with a uniform distribution on $[0,1]$. Moreover, it is assumed that each sensor can cover a range of length $1 / n$ to the left and to the right. In order to achieve full coverage, one has to move the sensors to the anchor positions $t_{i}$. This displacement incurs a cost which can be measured as

$$
\sum_{i=1}^{n}\left|X_{(i)}-t_{i}\right|^{a}
$$

where $X_{(i)}$ denotes the $i$-th order statistics of $X_{1}, \ldots, X_{n}$ and $a>0$ measures the effect of overcoming obstacles when moving sensors. Since the density of $X_{(i)}$ is given by

$$
f_{X_{(i)}}(x)=i\left(\begin{array}{c}
n \\
i
\end{array}\right) x^{i-1}(1-x)^{n-i}
$$

the expected value of $(1.3)$ is the above binomial sum.

Next, in the Poisson case, the $X_{i}$ are assumed to be the arrival times of a Poisson process on $[0, \infty)$ with rate $n$. The goal now is to place the $n$ sensors such that they are separated exactly by a distance of $1 / n$, i.e., $X_{i}$ should be placed at position $X_{1}+(i-1) / n$. The resulting displacement cost is given by

$$
\sum_{i=2}^{n}\left|X_{i}-X_{1}-s_{i-1}\right|^{a}=\sum_{i=0}^{n-2}\left|X_{i+2}-X_{1}-s_{i+1}\right|^{a}
$$

whose expected value is the above Poisson sum since the density of $X_{i+2}-X_{1}$ is given by

$$
f_{X_{i+2}-X_{1}}(x)=n e^{-n x} \frac{(n x)^{i}}{i !}
$$

The binomial sum (1.1) was analyzed in [6] where the authors derived the first-order asymptotics for $a>0$ being an even integer. For this, they used a combinatorial approach and this approach was extended in [5] to include also odd integers. Then, in [4], a probabilistic approach was given which allowed the author to find the first-order asymptotics for all $a>0$ by using the result from [6]. The final result given in [4] reads as follows.

Theorem 1.1. [4] As $n \rightarrow \infty$,

$$
S_{\mathrm{Bin}}(n)=\frac{\Gamma(a / 2+1)}{2^{a / 2}(a+1)} n^{1-a / 2}+\mathcal{O}\left(n^{-a / 2}\right) .
$$

As for the Poisson sum (1.2), a similar approach as in [4] was used in [3] (however, only the case of $a>0$ being an even integer was thoroughly treated in this paper). The final result in 3 reads as follows. 
Theorem 1.2. [3] As $n \rightarrow \infty$,

$$
S_{\text {Poi }}(n)=\frac{\Gamma(a+1)}{2^{a / 2} \Gamma(a / 2+2)} n^{1-a / 2}+\mathcal{O}\left(n^{-a / 2}\right) .
$$

The purpose of this paper is two-fold. First, we show that both of the results above can be derived by a unified approach based on the Laplace method (for an introduction into this method see, e.g., Appendix B in [2]). Thus, the two-stage approach from [3,4 based on a combination of combinatorial and probabilistic tools to derive the above two results is not really necessary. Moreover, using our approach, more terms in the asymptotic expansions could be derived as well. However, we will not pursue this further and instead show that full asymptotic expansions can be given when $a>0$ is an even integer. These full expansions even hold as identities. The proofs of this part will based on combinatorial tools such as generating functions. Finally, we will also discuss how one can obtain all the coefficients in these identities in a systematic way.

The paper is organized as follows. In the next section, we will explain that by using hand-waving, the above results quickly follow from the Laplace method. Then, in Section 3, we will present all the technical details for the heuristic arguments from Section 2. In Section 4, we will consider the case where $a>0$ is an even integer. Finally, Section 5 will contain some concluding remarks.

2. Asymptotics of $(1.1)$ and $(1.2)$ via the Laplace method: heuristics

We start with the binomial sum. The Laplace method will be applied to the sum of the integrand in (1.1), i.e.,

$$
\sum_{i=1}^{n}\left|x-t_{i}\right|^{a} i\left(\begin{array}{c}
n \\
i
\end{array}\right) x^{i-1}(1-x)^{n-i} .
$$

The application consists of several steps. First, the sum is broken into a major and minor range. Here, the major range comes from the range of validity of the local limit theorem of the binomial distribution which reads

$$
\left(\begin{array}{c}
n \\
i
\end{array}\right) x^{i}(1-x)^{n-i} \sim \frac{1}{\sqrt{2 \pi n x(1-x)}} e^{-t^{2} / 2},
$$

where $i=n x+t \sqrt{n x(1-x)}$ with $|t| \leq t_{n}^{*}$ (the cut-off point $t_{n}^{*}$ will be given below). Then, owning to the exponential small tails of the binomial distribution, we have

$$
\begin{aligned}
& \sum_{i=1}^{n}\left|x-t_{i}\right|^{a} i\left(\begin{array}{c}
n \\
i
\end{array}\right) x^{i-1}(1-x)^{n-i} \\
\sim & \sum_{t}\left|\frac{1}{2 n}-t \sqrt{\frac{x(1-x)}{n}}\right|^{a} \frac{n}{\sqrt{2 n \pi x(1-x)}} e^{-t^{2} / 2}
\end{aligned}
$$




$$
=(x(1-x))^{a / 2} n^{1-a / 2} \frac{1}{\sqrt{2 \pi n x(1-x)}} \sum_{t}\left|t-\frac{1}{2 \sqrt{n x(1-x)}}\right|^{a} e^{-t^{2} / 2} .
$$

Next, we replace the sum by an integral which gives

$$
\begin{aligned}
& \sum_{i=1}^{n}\left|x-t_{i}\right|^{a} i\left(\begin{array}{c}
n \\
i
\end{array}\right) x^{i-1}(1-x)^{n-i} \\
\sim & \frac{(x(1-x))^{a / 2} n^{1-a / 2}}{\sqrt{2 \pi}} \int_{-\infty}^{\infty}\left|t-\frac{1}{2 \sqrt{n x(1-x)}}\right|^{a} e^{-t^{2} / 2} \mathrm{~d} t \\
= & \frac{(x(1-x))^{a / 2} n^{1-a / 2}}{\sqrt{2 \pi}} \int_{-\infty}^{\infty}|u|^{a} e^{-(u+1 /(2 \sqrt{n x(1-x)}))^{2} / 2} \mathrm{~d} u \\
\sim & \frac{(x(1-x))^{a / 2} n^{1-a / 2}}{\sqrt{2 \pi}} \int_{-\infty}^{\infty}|u|^{a} e^{-u^{2} / 2} \mathrm{~d} u,
\end{aligned}
$$

where the second step follows by the change of variable $u=t-1 /(2 \sqrt{n x(1-x)})$ and the last step follows from the Maclaurin series of the exponential function and dropping remainder terms. Recall that

$$
\begin{aligned}
\frac{1}{\sqrt{2 \pi}} \int_{-\infty}^{\infty}|u|^{a} e^{-u^{2} / 2} \mathrm{~d} u & =\sqrt{\frac{2}{\pi}} \int_{0}^{\infty} u^{a} e^{-u^{2} / 2} \mathrm{~d} u \\
& =\frac{2^{a / 2}}{\sqrt{\pi}} \int_{0}^{\infty} v^{(a-1) / 2} e^{-v} \mathrm{~d} v=\frac{2^{a / 2}}{\sqrt{\pi}} \Gamma\left(\frac{a+1}{2}\right) .
\end{aligned}
$$

Thus, we obtain that

$$
\sum_{i=1}^{n}\left|x-t_{i}\right|^{a} i\left(\begin{array}{c}
n \\
i
\end{array}\right) x^{i-1}(1-x)^{n-i} \sim \frac{(x(1-x))^{a / 2} 2^{a / 2}}{\sqrt{\pi}} \Gamma\left(\frac{a+1}{2}\right) n^{1-a / 2} .
$$

Finally, in order to obtain (1.1), we have to integrate over $x$. For this, we will need that the above asymptotic expansion holds uniformly in a range of $x$ (which will turn out to be the case; see the next section). Then,

$$
\begin{aligned}
S_{\operatorname{Bin}}(n) & \sim \frac{2^{a / 2} \Gamma((a+1) / 2)}{\sqrt{\pi}}\left(\int_{0}^{1}(x(1-x))^{a / 2} \mathrm{~d} x\right) n^{1-a / 2} \\
& =\frac{2^{a / 2} \Gamma((a+1) / 2)}{\sqrt{\pi}} \beta(a / 2+1, a / 2+1) n^{1-a / 2} \\
& =\frac{2^{a / 2} \Gamma((a+1) / 2)}{\sqrt{\pi}} \cdot \frac{\Gamma^{2}(a / 2+1)}{\Gamma(a+2)} n^{1-a / 2},
\end{aligned}
$$

where $\beta(s, t)$ denotes the beta function. From this the claimed shape of the first-order term in Theorem 1.1 follows by using the duplication formula of the gamma function and straightforward computation. 
The proof of the expansion in Theorem 1.2 runs along similar lines. More precisely, we again apply the Laplace method to the sum of the integrand:

$$
\sum_{i=1}^{n}\left|x-s_{i+1}\right|^{a} n e^{-n x} \frac{(n x)^{i}}{i !} .
$$

As for the binomial sum, we break this sum into a major and minor range where the major range comes now from the range of validity of the local limit theorem for the Poisson distribution:

$$
e^{-n x} \frac{(n x)^{i}}{i !} \sim \frac{1}{\sqrt{2 \pi n x}} e^{-t^{2} / 2}
$$

where $i=n x+t \sqrt{n x}$ and $|t| \leq t_{n}^{*}$. Then, again by exponential small tails, we have

$$
\begin{aligned}
\sum_{i=1}^{n}\left|x-s_{i+1}\right|^{a} n e^{-n x} \frac{(n x)^{i}}{i !} & \sim \sum_{t}\left|t \sqrt{\frac{x}{n}}+\frac{1}{n}\right|^{a} \frac{n}{\sqrt{2 \pi n x}} e^{-t^{2}} \\
& =x^{a / 2} n^{1-a / 2} \frac{1}{\sqrt{2 \pi n x}} \sum_{t}\left|t+\frac{1}{\sqrt{x n}}\right|^{a} e^{-t^{2} / 2} .
\end{aligned}
$$

From this, by using the same arguments as above, we obtain that

$$
\sum_{i=1}^{n}\left|x-s_{i+1}\right|^{a} n e^{-n x} \frac{(n x)^{i}}{i !} \sim \frac{x^{a / 2} 2^{a / 2}}{\sqrt{\pi}} \Gamma\left(\frac{a+1}{2}\right) n^{1-a / 2}
$$

which again holds uniformly for some range in $x$; for details see the next section.

Finally, we have to integrate again over $x$ where it is sufficient to integrate from 0 to 1 since the tail will turn out to be negligible. This yields

$$
S_{\text {Poi }}(n) \sim \frac{2^{a / 2} \Gamma((a+1) / 2)}{\sqrt{\pi}}\left(\int_{0}^{1} x^{a / 2} \mathrm{~d} x\right) n^{1-a / 2}=\frac{2^{a / 2} \Gamma((a+1) / 2)}{(a / 2+1) \sqrt{\pi}} n^{1-a / 2}
$$

from which the claimed shape of the first-order term in Theorem 1.2 follows by another application of the duplication formula and straightforward computation.

3. Asymptotics of (1.1) and (1.2) via the Laplace method: details

In this section, we are going to fill in all details in the heuristic arguments from the previous section. We will break the section into two subsections. In the first, we will treat the binomial sum and in the second the Poisson sum.

\subsection{Asymptotics of the binomial sum}

We first recall the local limit theorem of the binomial distribution which follows straightforwardly from Stirling's approximation for $n !$. 
Proposition 3.1 (Local limit theorem for the binomial distribution). Let $0<p<1$. Then, for $i=n p+t \sqrt{n p(1-p)}$ and $t=o\left((n p(1-p))^{1 / 6}\right)$,

$$
\left(\begin{array}{c}
n \\
i
\end{array}\right) p^{i}(1-p)^{n-i}=\frac{e^{-t^{2} / 2}}{\sqrt{2 \pi n p(1-p)}}\left(1+\frac{p(t, p)}{\sqrt{n p(1-p)}}+\mathcal{O}\left(\frac{t^{6}+1}{n p(1-p)}\right)\right),
$$

where

$$
p(t, p)=(1-2 p)\left(\frac{t^{3}}{6}-\frac{t}{2}\right)
$$

and the implied constant in the $\mathcal{O}$-term is absolute.

Sketch of proof. Consider

$$
\left(\begin{array}{c}
n \\
i
\end{array}\right) p^{i}(1-p)^{n-i}=e^{\log (n !)-\log (i !)-\log ((n-i) !)+i \log p+(n-i) \log (1-p)} .
$$

From this the claim follows by using $i=n p+t \sqrt{n p(1-p)}$, Stirling's formula in the form

$$
n !=\left(\frac{n}{e}\right)^{n} \sqrt{2 \pi n}\left(1+\mathcal{O}\left(n^{-1}\right)\right)
$$

and Taylor series expansion.

Also, we recall a version of the Chernoff bound for the tail of the binomial distribution.

Proposition 3.2 (Chernoff bound). Let $X$ be binomial distributed random variable with parameters $(n, p)$. Then, for all $\delta \geq 1$,

$$
\mathbb{P}(X \geq(1+\delta) n p) \leq e^{-\delta n p / 3} .
$$

Proof. This follows, e.g., from Theorem 4.4 in 12 by using the inequality

$$
\log (1+\delta) \geq \frac{4 \delta}{3(1+\delta)}
$$

for $\delta \geq 1$.

Now, we can start with the proof. We first break the integral in 11.1 into three parts so that the local limit theorem can be applied to the middle part:

$$
\begin{aligned}
S_{\operatorname{Bin}}(n)= & \int_{0}^{1} \sum_{i=1}^{n} i\left(\begin{array}{c}
n \\
i
\end{array}\right)\left|x-t_{i}\right|^{a} x^{i-1}(1-x)^{n-i} \mathrm{~d} x \\
= & \int_{0}^{\rho_{n}} \sum_{i=1}^{n} i\left(\begin{array}{c}
n \\
i
\end{array}\right)\left|x-t_{i}\right|^{a} x^{i-1}(1-x)^{n-i} \mathrm{~d} x \\
& +\int_{\rho_{n}}^{1-\rho_{n}} \sum_{i=1}^{n} i\left(\begin{array}{c}
n \\
i
\end{array}\right)\left|x-t_{i}\right|^{a} x^{i-1}(1-x)^{n-i} \mathrm{~d} x \\
& +\int_{1-\rho_{n}}^{1} \sum_{i=1}^{n} i\left(\begin{array}{c}
n \\
i
\end{array}\right)\left|x-t_{i}\right|^{a} x^{i-1}(1-x)^{n-i} \mathrm{~d} x=: I_{1}+I_{2}+I_{3},
\end{aligned}
$$


where $\rho_{n}:=n^{-1+\epsilon}$ and $\epsilon>0$ will be chosen during the proof.

The Laplace method will be used to treat the integrand of $I_{2}$. Thus, according to the heuristic from the previous section, we have to break the sum into a major and minor range:

$$
\begin{aligned}
I_{2}= & \int_{\rho_{n}}^{1-\rho_{n}} \sum_{i=1}^{n} i\left(\begin{array}{c}
n \\
i
\end{array}\right)\left|x-t_{i}\right|^{a} x^{i-1}(1-x)^{n-i} \mathrm{~d} x \\
= & \int_{\rho_{n}}^{1-\rho_{n}} \sum_{|i-n x| \leq t_{n}^{*} \sqrt{n x(1-x)}} i\left(\begin{array}{c}
n \\
i
\end{array}\right)\left|x-t_{i}\right|^{a} x^{i-1}(1-x)^{n-i} \mathrm{~d} x \\
& +\int_{\rho_{n}}^{1-\rho_{n}} \sum_{|i-n x|>t_{n}^{*} \sqrt{n x(1-x)}} i\left(\begin{array}{c}
n \\
i
\end{array}\right)\left|x-t_{i}\right|^{a} x^{i-1}(1-x)^{n-i} \mathrm{~d} x=: I_{2,1}+I_{2,2},
\end{aligned}
$$

where $t_{n}^{*}=n^{\epsilon / 7}$.

The main contribution comes from $I_{2,1}$.

Lemma 3.3. We have

$$
I_{2,1}=\frac{\Gamma(a / 2+1)}{2^{a / 2}(a+1)} n^{1-a / 2}+\mathcal{O}\left(n^{-a / 2}\right) .
$$

Proof. By Proposition 3.1, we have

$$
\begin{aligned}
I_{2,1}= & \int_{\rho_{n}}^{1-\rho_{n}} \frac{(x(1-x))^{a / 2} n^{1-a / 2}}{\sqrt{2 \pi n x(1-x)}} \sum_{|i-n x| \leq t_{n}^{*} \sqrt{n x(1-x)}}\left|t-\frac{1}{2 \sqrt{n x(1-x)}}\right|^{a} e^{-t^{2} / 2} \\
& \quad \times\left(1+\frac{1}{\sqrt{n x(1-x)}}\left(\frac{t^{3}(1-2 x)}{6}+\frac{t}{2}\right)+\mathcal{O}\left(\frac{t^{6}+1}{n x(1-x)}\right)\right) \mathrm{d} x \\
= & I_{2,1,1}+I_{2,1,2}+I_{2,1,3},
\end{aligned}
$$

where $i=n x+t \sqrt{n x(1-x)}$ and the last three terms correspond to the three terms obtained by multiplying the integral with the terms in the bracket.

For $I_{2,1,1}$, we approximate the sum by an integral which yields an exponential small error term (however, we will use $\mathcal{O}\left(n^{-a / 2}\right)$ since this will turn out to be the second order term):

$$
I_{2,1,1}=\int_{\rho_{n}}^{1-\rho_{n}} \frac{(x(1-x))^{a / 2} n^{1-a / 2}}{\sqrt{2 \pi}} \int_{-t_{n}^{*}}^{t_{n}^{*}}\left|t-\frac{1}{2 \sqrt{n x(1-x)}}\right|^{a} e^{-t^{2} / 2} \mathrm{~d} t \mathrm{~d} x+\mathcal{O}\left(n^{-a / 2}\right) .
$$

For the inner integral, as explained in the previous section, we use the change of variable

$$
u=t-\frac{1}{2 \sqrt{n x(1-x)}}
$$


and then the Maclaurin series expansion of the exponential function. This gives

$$
\begin{aligned}
& \frac{1}{\sqrt{2 \pi}} \int_{-t_{n}^{*}}^{t_{n}^{*}}\left|t-\frac{1}{2 \sqrt{n x(1-x)}}\right|^{a} e^{-t^{2} / 2} \mathrm{~d} t \\
= & \frac{1}{\sqrt{2 \pi}} \int_{-t_{n}^{*}-1 /(2 \sqrt{n x(1-x)})}^{t_{n}^{*}-1 /(2 \sqrt{n x(1-x)})}|u|^{a} e^{-(u+1 /(2 \sqrt{n x(1-x)}))^{2} / 2} \mathrm{~d} u \\
= & \frac{1}{\sqrt{2 \pi}} \int_{-t_{n}^{*}-1 /(2 \sqrt{n x(1-x)})}^{t_{n}^{*}-1 /(2 \sqrt{n x(1-x)})}|u|^{a} e^{-u^{2} / 2}\left(1-\frac{u}{2 \sqrt{n x(1-x)}}+\mathcal{O}\left(\frac{u^{2}+1}{n x(1-x)}\right)\right) \mathrm{d} u \\
= & \frac{1}{\sqrt{2 \pi}} \int_{-\infty}^{\infty}|u|^{a} e^{-u^{2} / 2}\left(1-\frac{u}{2 \sqrt{n x(1-x)}}+\mathcal{O}\left(\frac{u^{2}+1}{n x(1-x)}\right)\right) \mathrm{d} u+\mathcal{O}\left(n^{-1}\right),
\end{aligned}
$$

where in the last step we attached the tails which contribute the error bound:

$$
\begin{aligned}
& \frac{1}{\sqrt{2 \pi}} \int_{t_{n}^{*}-1 /(2 \sqrt{n x(1-x)})}^{\infty} u^{a} e^{-u^{2} / 2}\left(1-\frac{u}{2 \sqrt{n x(1-x)}}+\mathcal{O}\left(\frac{u^{2}+1}{n x(1-x)}\right)\right) \mathrm{d} u \\
= & \mathcal{O}\left(e^{-c n^{2 \epsilon / 7}}\right)=\mathcal{O}\left(n^{-1}\right),
\end{aligned}
$$

where $c>0$ is a suitable constant. A similar bound also holds for the other tail.

Next, recall from 2.1,

$$
\frac{1}{\sqrt{2 \pi}} \int_{-\infty}^{\infty}|u|^{a} e^{-u^{2} / 2} \mathrm{~d} u=\frac{2^{a / 2}}{\sqrt{\pi}} \Gamma\left(\frac{a+1}{2}\right)
$$

and trivially

$$
\int_{-\infty}^{\infty} u|u|^{a} e^{-u^{2} / 2} \mathrm{~d} u=0
$$

Plugging this into 3.3 gives

$$
\frac{1}{\sqrt{2 \pi}} \int_{-t_{n}^{*}}^{t_{n}^{*}}\left|t-\frac{1}{2 \sqrt{n x(1-x)}}\right|^{a} e^{-t^{2} / 2} \mathrm{~d} t=\frac{2^{a / 2}}{\sqrt{\pi}} \Gamma\left(\frac{a+1}{2}\right)+\mathcal{O}\left(\frac{1}{n x(1-x)}\right)+\mathcal{O}\left(n^{-1}\right) .
$$

Now, we plug this in turn into $(3.2)$ and obtain that

$$
\begin{aligned}
I_{2,1,1} & =\frac{2^{a / 2} \Gamma((a+1) / 2)}{\sqrt{\pi}}\left(\int_{\rho_{n}}^{1-\rho_{n}}(x(1-x))^{a / 2} \mathrm{~d} x\right) n^{1-a / 2}+\mathcal{O}\left(n^{-a / 2}\right) \\
& =\frac{2^{a / 2} \Gamma((a+1) / 2)}{\sqrt{\pi}}\left(\int_{0}^{1}(x(1-x))^{a / 2} \mathrm{~d} x\right) n^{1-a / 2}+\mathcal{O}\left(n^{-a(1-\epsilon / 2)+\epsilon}\right)+\mathcal{O}\left(n^{-a / 2}\right),
\end{aligned}
$$

where the first error term is smaller than the second by choosing $\epsilon>0$ small enough. Finally, by the same computation as in the last section, we obtain that

$$
I_{2,1,1}=\frac{\Gamma(a / 2+1)}{2^{a / 2}(a+1)} n^{1-a / 2}+\mathcal{O}\left(n^{-a / 2}\right) .
$$


Next, we consider $I_{2,1,2}$ for which the same line of reasoning as above yields

$$
I_{2,1,2}=\mathcal{O}\left(n^{-a / 2}\right)
$$

Note that an important point here is that the function in $t$ of the second term inside the bracket in (3.1) is odd and thus a similar cancellation as for the second term inside the bracket of (3.3) takes place which causes the error to be of the claimed size (and not of the size $\left.\mathcal{O}\left(n^{(1-a) / 2}\right)\right)$.

Finally, for $I_{2,1,3}$, we have the simple bound

$$
I_{2,1,3}=\mathcal{O}\left(n^{-a / 2} \int_{0}^{1}(x(1-x))^{a / 2-1} \mathrm{~d} x\right)=\mathcal{O}\left(n^{-a / 2}\right) .
$$

Collecting all three bounds gives the final result for $I_{2,1}$.

Next, we show that $I_{2,2}$ is negligible. (This was also one of the claims of the last section.)

Lemma 3.4. We have

$$
I_{2,2}=\mathcal{O}\left(n^{-a / 2}\right) .
$$

Proof. The crucial observation is that

$$
\left(\begin{array}{c}
n \\
i
\end{array}\right) x^{i}(1-x)^{n-i}=\mathcal{O}\left(e^{-c n^{2 \epsilon / 7}}\right)
$$

uniformly for all $i$ with $|i-n x|>t_{n}^{*} \sqrt{n x(1-x)}$ and $x$ with $\rho_{n} \leq x \leq 1-\rho_{n}$ where $c>0$ is a suitable constant. This follows from the fact that the binomial coefficients for this range of $i$ are bounded by the ones with either

$$
i=n x+t_{n}^{*} \sqrt{n x(1-x)}+\mathcal{O}(1) \quad \text { or } \quad i=n x-t_{n}^{*} \sqrt{n x(1-x)}+\mathcal{O}(1)
$$

which are of the above claimed order.

Using the above estimate, we now obtain that

$$
\begin{aligned}
I_{2,2} & =\int_{\rho_{n}}^{1-\rho_{n}} \sum_{|i-n x|>t_{n}^{*} \sqrt{n x(1-x)}} i\left(\begin{array}{c}
n \\
i
\end{array}\right)\left|x-t_{i}\right|^{a} x^{i-1}(1-x)^{n-i} \mathrm{~d} x \\
& =\mathcal{O}\left(n^{2} e^{-c n^{2 \epsilon / 7}}\right)=\mathcal{O}\left(n^{-a / 2}\right) .
\end{aligned}
$$

This shows the claimed bound.

Putting the estimate in the last two lemmas together, we obtain the following corollary.

Corollary 3.5. We have

$$
I_{2}=\frac{\Gamma(a / 2+1)}{2^{a / 2}(a+1)} n^{1-a / 2}+\mathcal{O}\left(n^{-a / 2}\right) .
$$


What is left is to show that $I_{1}$ and $I_{3}$ are negligible. We will give the details only for $I_{1}$ since $I_{3}$ can be treated similarly due to symmetry.

We will break $I_{1}$ again into two parts according to the major and minor range of the binomial distribution:

$$
\begin{aligned}
I_{1}= & \int_{0}^{\rho_{n}} \sum_{i=1}^{n} i\left(\begin{array}{c}
n \\
i
\end{array}\right)\left|x-t_{i}\right|^{a} x^{i-1}(1-x)^{n-i} \mathrm{~d} x \\
= & \int_{0}^{\rho_{n}} \sum_{i \leq 2 n^{\epsilon}} i\left(\begin{array}{c}
n \\
i
\end{array}\right)\left|x-t_{i}\right|^{a} x^{i-1}(1-x)^{n-i} \mathrm{~d} x \\
& +\int_{0}^{\rho_{n}} \sum_{i>2 n^{\epsilon}} i\left(\begin{array}{c}
n \\
i
\end{array}\right)\left|x-t_{i}\right|^{a} x^{i-1}(1-x)^{n-i} \mathrm{~d} x \\
= & : I_{1,1}+I_{1,2} .
\end{aligned}
$$

We start with bounding $I_{1,1}$.

Lemma 3.6. We have

$$
I_{1,1}=\mathcal{O}\left(n^{-a / 2}\right)
$$

Proof. First, note that for $1 \leq i \leq 2 n^{\epsilon}$ and $0 \leq x \leq \rho_{n}$,

$$
\left|x-t_{i}\right|=\left|x-\frac{i}{n}+\frac{1}{2 n}\right|=\mathcal{O}\left(n^{-1+\epsilon}\right) .
$$

Also, recall that

$$
n x=\sum_{i=0}^{n} i\left(\begin{array}{c}
n \\
i
\end{array}\right) x^{i}(1-x)^{n-i} .
$$

Thus,

$$
\begin{aligned}
I_{1,1} & =\int_{0}^{\rho_{n}} \sum_{i \leq 2 n^{\epsilon}} i\left(\begin{array}{c}
n \\
i
\end{array}\right)\left|x-t_{i}\right|^{a} x^{i-1}(1-x)^{n-i} \mathrm{~d} x \\
& =\mathcal{O}\left(n^{-a+a \epsilon} \int_{0}^{\rho_{n}} \frac{1}{x} \sum_{i \leq 2 n^{\epsilon}} i\left(\begin{array}{c}
n \\
i
\end{array}\right) x^{i}(1-x)^{n-i} \mathrm{~d} x\right) \\
& =\mathcal{O}\left(n^{-a+a \epsilon+\epsilon}\right)=\mathcal{O}\left(n^{-a / 2}\right),
\end{aligned}
$$

where the last step holds if $\epsilon$ is chosen sufficiently small.

Next, we have to bound $I_{1,2}$.

Lemma 3.7. We have

$$
I_{1,2}=\mathcal{O}\left(n^{-a / 2}\right) .
$$


Proof. We again break the integral into two parts:

$$
\begin{aligned}
I_{1,2}= & \int_{0}^{\rho_{n}} \sum_{i>2 n^{\epsilon}} i\left(\begin{array}{c}
n \\
i
\end{array}\right)\left|x-t_{i}\right|^{a} x^{i-1}(1-x)^{n-i} \mathrm{~d} x \\
= & \int_{0}^{n^{-a / 2-1}} \sum_{i>2 n^{\epsilon}} i\left(\begin{array}{c}
n \\
i
\end{array}\right)\left|x-t_{i}\right|^{a} x^{i-1}(1-x)^{n-i} \mathrm{~d} x \\
& +\int_{n^{-a / 2-1}}^{\rho_{n}} \sum_{i>2 n^{\epsilon}} i\left(\begin{array}{c}
n \\
i
\end{array}\right)\left|x-t_{i}\right|^{a} x^{i-1}(1-x)^{n-i} \mathrm{~d} x=: I_{1,2,1}+I_{1,2,2} .
\end{aligned}
$$

For $I_{1,2,1}$, we use $\left|x-t_{i}\right|=\mathcal{O}(1)$ and thus

$$
\begin{aligned}
I_{1,2,1} & =\int_{0}^{n^{-a / 2-1}} \sum_{i>2 n^{\epsilon}} i\left(\begin{array}{c}
n \\
i
\end{array}\right)\left|x-t_{i}\right|^{a} x^{i-1}(1-x)^{n-i} \mathrm{~d} x \\
& =\mathcal{O}\left(\int_{0}^{n^{-a / 2-1}} \frac{1}{x} \sum_{i=1}^{n} i\left(\begin{array}{c}
n \\
i
\end{array}\right) x^{i}(1-x)^{n-i} \mathrm{~d} x\right)=\mathcal{O}\left(n^{-a / 2}\right) .
\end{aligned}
$$

For $I_{1,2,2}$, we use Proposition 3.2 which implies

$$
\sum_{i>2 n^{\epsilon}}\left(\begin{array}{c}
n \\
i
\end{array}\right) x^{i}(1-x)^{n-i}=\mathcal{O}\left(e^{-n^{\epsilon} / 3}\right) .
$$

Thus,

$$
\begin{aligned}
I_{1,2,2} & =\int_{n^{-a / 2-1}}^{\rho_{n}} \sum_{i>2 n^{\epsilon}} i\left(\begin{array}{c}
n \\
i
\end{array}\right)\left|x-t_{i}\right|^{a} x^{i-1}(1-x)^{n-i} \mathrm{~d} x \\
& =\mathcal{O}\left(n e^{-n^{\epsilon} / 3} \int_{n^{-a / 2-1}}^{\rho_{n}} \frac{1}{x} \mathrm{~d} x\right)=\mathcal{O}\left(\log (n) n e^{-n^{\epsilon} / 3}\right)=\mathcal{O}\left(n^{-a / 2}\right) .
\end{aligned}
$$

Combining the above two bounds gives the claimed result.

As a corollary from the last two lemmas, we obtain the following.

Corollary 3.8. We have

$$
I_{1}=\mathcal{O}\left(n^{-a / 2}\right) \quad \text { and } \quad I_{3}=\mathcal{O}\left(n^{-a / 2}\right) .
$$

This corollary and the one above now immediately imply Theorem 1.1 .

\subsection{Asymptotics of the Poisson sum}

In this section, we treat the Poisson sum 1.2 .

As for the binomial sum, an important tool will be the local limit theorem (which follows with the same method of proof as Proposition 3.1. 
Proposition 3.9 (Local limit theorem for the Poisson distribution). Let $\lambda>0$. Then, for $i=\lambda+t \sqrt{\lambda}$ and $t=o\left(\lambda^{1 / 6}\right)$,

$$
e^{-\lambda} \frac{\lambda^{i}}{i !}=\frac{e^{-t^{2} / 2}}{\sqrt{2 \pi \lambda}}\left(1+\frac{1}{\sqrt{\lambda}}\left(\frac{t^{3}}{6}-\frac{t}{2}\right)+\mathcal{O}\left(\frac{t^{6}+1}{\lambda}\right)\right),
$$

where the implied constant in the $\mathcal{O}$-term is absolute.

Also, we need the following tail bounds for the Poisson distribution; see [1].

Proposition 3.10. Let $X$ be a Poisson distributed random variable with parameter $\lambda$. Then, for all $t>0$,

$$
\mathbb{P}(X \geq \lambda+t) \leq e^{-t^{2} /(2 \lambda+2 t)}
$$

and for all $0<t<\lambda$,

$$
\mathbb{P}(X \leq \lambda-t) \leq e^{-t^{2} /(2 \lambda+2 t)} .
$$

Now, we can start with the proof of Theorem 1.2 . We first break the integral in 1.2 into three parts:

$$
\begin{aligned}
S_{\text {Poi }}(n)= & \int_{0}^{\infty} \sum_{i=0}^{n-2}\left|x-s_{i+1}\right|^{a} n e^{-n x} \frac{(n x)^{i}}{i !} \mathrm{d} x \\
= & \int_{0}^{\rho_{n}} \sum_{i=0}^{n-2}\left|x-s_{i+1}\right|^{a} n e^{-n x} \frac{(n x)^{i}}{i !} \mathrm{d} x+\int_{\rho_{n}}^{2} \sum_{i=0}^{n-2}\left|x-s_{i+1}\right|^{a} n e^{-n x} \frac{(n x)^{i}}{i !} \mathrm{d} x \\
& +\int_{2}^{\infty} \sum_{i=0}^{n-2}\left|x-s_{i+1}\right|^{a} n e^{-n x} \frac{(n x)^{i}}{i !} \mathrm{d} x=: I_{1}+I_{2}+I_{3},
\end{aligned}
$$

where $\rho_{n}:=n^{-1+\epsilon}$ and $\epsilon>0$ will be chosen below.

As for the binomial sum, we will apply the Laplace method to the integrand of $I_{2}$ which we break into two parts:

$$
\begin{aligned}
I_{2}= & \int_{\rho_{n}}^{2} \sum_{i=0}^{n-2}\left|x-s_{i+1}\right|^{a} n e^{-n x} \frac{(n x)^{i}}{i !} \mathrm{d} x \\
= & \int_{\rho_{n}}^{2} \sum_{\substack{|i-n x| \leq t_{n}^{*} \sqrt{n x} \\
i \leq n-2}}\left|x-s_{i+1}\right|^{a} n e^{-n x} \frac{(n x)^{i}}{i !} \mathrm{d} x \\
& +\int_{\rho_{n}}^{2} \sum_{\substack{|i-n x|>t_{n}^{*} \sqrt{n x} \\
i \leq n-2}}\left|x-s_{i+1}\right|^{a} n e^{-n x} \frac{(n x)^{i}}{i !} \mathrm{d} x=: I_{2,1}+I_{2,2},
\end{aligned}
$$

where $t_{n}^{*}=n^{\epsilon / 7}$.

Theorem 1.2 will now follow from the following series of results. 
Lemma 3.11. We have

$$
I_{2,1}=\frac{\Gamma(a+1)}{2^{a / 2} \Gamma(a / 2+2)} n^{1-a / 2}+\mathcal{O}\left(n^{-a / 2}\right) .
$$

Proof. We first plug into the expression for $I_{2,1}$ the local limit theorem from Proposition 3.9 :

$$
\begin{aligned}
I_{2,1}= & \int_{\rho_{n}}^{2} \frac{x^{a / 2} n^{1-a / 2}}{\sqrt{2 \pi n x}} \sum_{\substack{|i-n x| \leq t_{n}^{*} \sqrt{n x} \\
i \leq n-2}}\left|t+\frac{1}{\sqrt{n x}}\right|^{a} e^{-t^{2} / 2} \\
& \times\left(1+\frac{1}{\sqrt{n x}} \frac{t\left(3-t^{2}\right)}{6}+\mathcal{O}\left(\frac{t^{6}+1}{n x}\right)\right) \mathrm{d} x .
\end{aligned}
$$

This expression will be broken into three parts according to the three terms in the bracket inside the integrand.

We will only treat in details the first part; the other two can be handled by similar tools; see also the proof of Lemma 3.3 .

First, by replacing the sum by an integral, we have

$$
\begin{aligned}
& \int_{\rho_{n}}^{2} \frac{x^{a / 2} n^{1-a / 2}}{\sqrt{2 \pi n x}} \sum_{\substack{|i-n x| \leq t_{n}^{*} \sqrt{n x} \\
i \leq n-2}} x^{a / 2}\left|t+\frac{1}{\sqrt{n x}}\right|^{a} e^{-t^{2} / 2} \mathrm{~d} x \\
= & \int_{\rho_{n}}^{2} \frac{x^{a / 2} n^{1-a / 2}}{\sqrt{2 \pi}} \int_{-t_{n}^{*}}^{\alpha(n ; x)}\left|t+\frac{1}{\sqrt{n x}}\right|^{a} e^{-t^{2} / 2} \mathrm{~d} t \mathrm{~d} x+\mathcal{O}\left(n^{-a / 2}\right),
\end{aligned}
$$

where the error term in fact is exponentially small and

$$
\alpha(n ; x)=\frac{\sqrt{n}(1-x)}{\sqrt{x}}
$$

if, for $\rho_{n} \leq x \leq 2$, this value is between $-t_{n}^{*}$ and $t_{n}^{*}$ or one of the boundary points otherwise.

Now, we make the substitution $u=t+1 / \sqrt{n x}$ which gives

$$
\begin{aligned}
& \int_{\rho_{n}}^{2} \frac{x^{a / 2} n^{1-a / 2}}{\sqrt{2 \pi}} \int_{-t_{n}^{*}}^{\alpha(n ; x)}\left|t+\frac{1}{\sqrt{n x}}\right|^{a} e^{-t^{2} / 2} \mathrm{~d} t \mathrm{~d} x+\mathcal{O}\left(n^{-a / 2}\right) \\
= & \int_{\rho_{n}}^{2} \frac{x^{a / 2} n^{1-a / 2}}{\sqrt{2 \pi}} \int_{-t_{n}^{*}+1 / \sqrt{n x}}^{\alpha(n ; x)+1 / \sqrt{n x}}|u|^{a} e^{-(u-1 / \sqrt{n x})^{2} / 2} \mathrm{~d} u \mathrm{~d} x+\mathcal{O}\left(n^{-a / 2}\right) \\
= & \int_{\rho_{n}}^{2} \frac{x^{a / 2} n^{1-a / 2}}{\sqrt{2 \pi}} \int_{-t_{n}^{*}+1 / \sqrt{n x}}^{\alpha(n ; x)+1 / \sqrt{n x}}|u|^{a} e^{-u^{2} / 2}\left(1+\frac{u}{\sqrt{n x}}+\mathcal{O}\left(\frac{u^{2}+1}{n x}\right)\right) \mathrm{d} u \mathrm{~d} x+\mathcal{O}\left(n^{-a / 2}\right) .
\end{aligned}
$$

Next, we switch the order of integration which yields

$$
\frac{n^{1-a / 2}}{\sqrt{2 \pi}} \int_{\rho_{n}}^{2} \int_{-t_{n}^{*}+1 / \sqrt{n x}}^{\alpha(n ; x)+1 / \sqrt{n x}} x^{a / 2}|u|^{a} e^{-u^{2} / 2}\left(1+\frac{u}{\sqrt{n x}}+\mathcal{O}\left(\frac{u^{2}+1}{n x}\right)\right) \mathrm{d} u \mathrm{~d} x+\mathcal{O}\left(n^{-a / 2}\right)
$$




$$
\begin{aligned}
= & \frac{n^{1-a / 2}}{\sqrt{2 \pi}} \int_{-t_{n}^{*}+1 / \sqrt{2 n}}^{t_{n}^{*}+n^{-\epsilon / 2}} \int_{\rho_{n}}^{\beta(n ; u)} x^{a / 2}|u|^{a} e^{-u^{2} / 2}\left(1+\frac{u}{\sqrt{n x}}+\mathcal{O}\left(\frac{u^{2}+1}{n x}\right)\right) \mathrm{d} x \mathrm{~d} u \\
& +\mathcal{O}\left(n^{-a / 2}\right),
\end{aligned}
$$

where

$$
\beta(n ; u)=1+\frac{u \sqrt{4 n+u^{2}+4}}{2 n}+\frac{u^{2}+2}{2 n}=1+\frac{u}{\sqrt{n}}+\mathcal{O}\left(\frac{u^{2}+1}{n}\right) .
$$

Carrying out the integration with expect to $x$ gives:

$$
\begin{aligned}
& \frac{n^{1-a / 2}}{\sqrt{2 \pi}} \int_{-t_{n}^{*}+1 / \sqrt{2 n}}^{t_{n}^{*}+n^{-\epsilon / 2}} \int_{\rho_{n}}^{\beta(n ; u)} x^{a / 2}|u|^{a} e^{-u^{2} / 2}\left(1+\frac{u}{\sqrt{n x}}+\mathcal{O}\left(\frac{u^{2}+1}{n x}\right)\right) \mathrm{d} x \mathrm{~d} u+\mathcal{O}\left(n^{-a / 2}\right) \\
= & \frac{n^{1-a / 2}}{(a / 2+1) \sqrt{2 \pi}} \int_{-t_{n}^{*}+1 / \sqrt{2 n}}^{t_{n}^{*}+n^{-\epsilon / 2}}|u|^{a} e^{-u^{2} / 2}\left(1+c \frac{u}{\sqrt{n}}+\mathcal{O}\left(\frac{u^{2}+1}{n}\right)\right) \mathrm{d} u+\mathcal{O}\left(n^{-a / 2}\right),
\end{aligned}
$$

where $c=2+a / 2+1 /(a+1)$.

Now, for the rest of the proof, we can proceed as in Lemma 3.3. First, we attach the tails of the integral (which gives an exponentially small error) and then we evaluate the integral (which causes the second term in the bracket to disappear because the coefficient is an odd function in $u$ ). This yields

$$
\begin{aligned}
& \frac{n^{1-a / 2}}{(a / 2+1) \sqrt{2 \pi}} \int_{-t_{n}^{*}+1 / \sqrt{2 n}}^{t_{n}^{*}+n^{-\epsilon / 2}}|u|^{a} e^{-u^{2} / 2}\left(1+c \frac{u}{\sqrt{n}}+\mathcal{O}\left(\frac{u^{2}+1}{n}\right)\right) \mathrm{d} u+\mathcal{O}\left(n^{-a / 2}\right) \\
= & \frac{2^{a / 2} \Gamma((a+1) / 2)}{(a / 2+1) \sqrt{\pi}} n^{1-a / 2}+\mathcal{O}\left(n^{-a / 2}\right) .
\end{aligned}
$$

From this the claim follows as explained in the heuristic from the previous section.

Lemma 3.12. We have

$$
I_{2,2}=\mathcal{O}\left(n^{-a / 2}\right)
$$

Proof. The proof is similar to the proof of Lemma 3.4. More precisely, one only has to replace (3.4) by

$$
e^{-n x} \frac{(n x)^{i}}{i !}=\mathcal{O}\left(e^{-c n^{2 \epsilon / 7}}\right)
$$

which again holds uniformly for $|i-n x|>t_{n}^{*} \sqrt{n x}$ and all $x \geq \rho_{n}$ since all the terms are again bounded by the two boundary cases:

$$
i=n x+t_{n}^{*} \sqrt{n x}+\mathcal{O}(1) \quad \text { or } \quad i=n x-t_{n}^{*} \sqrt{n x}+\mathcal{O}(1) .
$$

Thus,

$$
I_{2,2}=\int_{\rho_{n}}^{2} \sum_{\substack{|i-n x|>t_{n}^{*} \sqrt{n x} \\ i \leq n-2}}\left|x-s_{i+1}\right|^{a} n e^{-n x} \frac{(n x)^{i}}{i !} \mathrm{d} x=\mathcal{O}\left(n^{2} e^{-c n^{2 \epsilon / 7}}\right)=\mathcal{O}\left(n^{-a / 2}\right)
$$

as claimed. 
From the last two lemmas, we have the following corollary.

Corollary 3.13. We have

$$
I_{2}=\frac{\Gamma(a+1)}{2^{a / 2} \Gamma(a / 2+2)} n^{1-a / 2}+\mathcal{O}\left(n^{-a / 2}\right) .
$$

Now, what is left is to prove that $I_{1}$ and $I_{3}$ are negligible.

Lemma 3.14. We have

$$
I_{1}=\mathcal{O}\left(n^{-a / 2}\right)
$$

Proof. As for the binomial sum above, we break $I_{1}$ into two parts:

$$
\begin{aligned}
I_{1} & =\int_{0}^{\rho_{n}} \sum_{i=0}^{n-2}\left|x-s_{i+1}\right|^{a} n e^{-n x} \frac{(n x)^{i}}{i !} \mathrm{d} x \\
& =\int_{0}^{\rho_{n}} \sum_{i \leq 2 n^{\epsilon}}\left|x-s_{i+1}\right|^{a} n e^{-n x} \frac{(n x)^{i}}{i !} \mathrm{d} x+\int_{0}^{\rho_{n}} \sum_{2 n^{\epsilon}<i \leq n-2}\left|x-s_{i+1}\right|^{a} n e^{-n x} \frac{(n x)^{i}}{i !} \mathrm{d} x \\
& =: I_{1,1}+I_{1,2} .
\end{aligned}
$$

For $I_{1,1}$, as in Lemma 3.6, we obtain that

$$
I_{1,1}=\int_{0}^{\rho_{n}} \sum_{i \leq 2 n^{\epsilon}}\left|x-s_{i+1}\right|^{a} n e^{-n x} \frac{(n x)^{i}}{i !} \mathrm{d} x=\mathcal{O}\left(n^{-a+a \epsilon+\epsilon}\right)=\mathcal{O}\left(n^{-a / 2}\right)
$$

by choosing $\epsilon>0$ small enough.

For $I_{1,2}$, by Proposition 3.10 .

$$
\sum_{i>2 n^{\epsilon}} e^{-n x} \frac{(n x)^{i}}{i !} \leq \sum_{i \geq n x+n^{\epsilon}} e^{-n x} \frac{(n x)^{i}}{i !} \leq e^{-n^{\epsilon} / 4},
$$

where $0 \leq x \leq \rho_{n}$. Consequently, since $\left|x-s_{i+1}\right|=\mathcal{O}(1)$,

$$
I_{1,2}=\int_{0}^{\rho_{n}} \sum_{2 n^{\epsilon}<i \leq n-2}\left|x-s_{i+1}\right|^{a} n e^{-n x} \frac{(n x)^{i}}{i !} \mathrm{d} x=\mathcal{O}\left(n e^{-n^{\epsilon} / 4}\right)=\mathcal{O}\left(n^{-a / 2}\right)
$$

which gives the claimed bound also for the second part.

Lemma 3.15. We have

$$
I_{3}=\mathcal{O}\left(n^{-a / 2}\right)
$$

Proof. From Proposition 3.10 , we obtain that

$$
\sum_{i=0}^{n-2} e^{-n x} \frac{(n x)^{i}}{i !} \leq \sum_{i=0}^{(n x) / 2} e^{-n x} \frac{(n x)^{i}}{i !} \leq e^{-(n x) / 12},
$$

where $x \geq 2$. Consequently, since $\left|x-s_{i+1}\right|=\mathcal{O}(x)$ for $x \geq 2$, we have

$$
I_{3}=\int_{2}^{\infty} \sum_{i=0}^{n-2}\left|x-s_{i+1}\right|^{a} n e^{-n x} \frac{(n x)^{i}}{i !} \mathrm{d} x=\mathcal{O}\left(n \int_{2}^{\infty} x^{a} e^{-(n x) / 12} \mathrm{~d} x\right)=\mathcal{O}\left(n^{-a / 2}\right)
$$

which is the claimed result. 


\section{The case of $a$ an even integer}

In this section, we will consider (1.1) and (1.2) when $a>0$ is an even integer. This case was also considered separately in [3, 6] where it was used in order to prove Theorems 1.1 and 1.2 .

The method used in 3,6 was combinatorial. We will use in this section also a combinatorial approach which is (i) structurally simpler than the one in [3,6] and (ii) reveals full asymptotic expansions which hold as identities and whose coefficients can be computed in a systematic way. (In fact, these identities are already implicitly contained in [3,6], where however the results were only stated as first-order asymptotic results.)

We will again break the section into two subsections according to whether the binomial sum or the Poisson sum is considered.

\subsection{Binomial sum for $a$ an even integer}

We first consider the right-hand side of (1.1) with the absolute value dropped:

$$
\begin{aligned}
M_{a}(n) & :=\sum_{i=0}^{n} \int_{0}^{1}\left(x-t_{i}\right)^{a} i\left(\begin{array}{c}
n \\
i
\end{array}\right) x^{i-1}(1-x)^{n-i} \mathrm{~d} x \\
& =\sum_{i=0}^{n} i\left(\begin{array}{c}
n \\
i
\end{array}\right) \int_{0}^{1}\left(x-\frac{i}{n}+\frac{1}{2 n}\right)^{a} x^{i-1}(1-x)^{n-i} \mathrm{~d} x,
\end{aligned}
$$

where $a$ is an integer which from now on is assumed to be $a \geq 0$.

Note that this sum corresponds to 1.1 when $a$ is an even integer and vanishes when $a$ is an odd integer.

Lemma 4.1. For odd $a$, we have $M_{a}(n)=0$.

Proof. Replacing $x$ by $1-x$ and $i$ by $n-i+1$ in the definition of $M_{a}(n)$ gives

$$
\begin{aligned}
M_{a}(n)= & \sum_{i=0}^{n}(n-i+1)\left(\begin{array}{c}
n \\
n-i+1
\end{array}\right) \\
& \times \int_{0}^{1}\left((1-x)-\frac{n-i+1}{n}+\frac{1}{2 n}\right)^{a}(1-x)^{(n-i+1)-1} x^{n-(n-i+1)} \mathrm{d} x \\
= & \sum_{i=0}^{n} i\left(\begin{array}{c}
n \\
i
\end{array}\right) \int_{0}^{1}\left(-x+\frac{i}{n}-\frac{1}{2 n}\right)^{a}(1-x)^{n-i} x^{i-1} \mathrm{~d} x \\
= & \sum_{i=0}^{n} i\left(\begin{array}{c}
n \\
i
\end{array}\right) \int_{0}^{1}(-1)^{a}\left(x-\frac{i}{n}+\frac{1}{2 n}\right)^{a}(1-x)^{n-i} x^{i-1} \mathrm{~d} x \\
= & (-1)^{a} M_{a}(n) .
\end{aligned}
$$

From this the claim follows. 
For general $a$, we consider the exponential-generating function:

$$
f_{n}(z):=\frac{1}{n} \sum_{a \geq 0} M_{a}(n) \frac{z^{a}}{a !},
$$

where the pre-factor $1 / n$ is used to simplify resulting expressions.

For this function, we have the following lemma.

Lemma 4.2. We have

$$
f_{n}(n z)=\frac{z / 2}{\sinh (z / 2)} \int_{0}^{1}\left((1-x) e^{x z}+x e^{-(1-x) z}\right)^{n} \mathrm{~d} x .
$$

Proof. Consider

$$
\begin{aligned}
f_{n}(z) & =\sum_{a \geq 0} \sum_{i=0}^{n} \int_{0}^{1} \frac{\left(z\left(x-\frac{i}{n}+\frac{1}{2 n}\right)\right)^{a}}{a !} \frac{i}{n}\left(\begin{array}{c}
n \\
i
\end{array}\right) x^{i-1}(1-x)^{n-i} \mathrm{~d} x \\
& =\int_{0}^{1} \sum_{i=1}^{n} e^{\left(x-\frac{i}{n}+\frac{1}{2 n}\right) z\left(\begin{array}{c}
n-1 \\
i-1
\end{array}\right) x^{i-1}(1-x)^{n-i} \mathrm{~d} x} \\
& =\int_{0}^{1} e^{\left(x-\frac{1}{2 n}\right) z} \sum_{i=1}^{n}\left(\begin{array}{c}
n-1 \\
i-1
\end{array}\right)\left(x e^{-z / n}\right)^{i-1}(1-x)^{n-i} \mathrm{~d} x \\
& =\int_{0}^{1} e^{\left(x-\frac{1}{2 n}\right) z}\left(1-x+x e^{-z / n}\right)^{n-1} \mathrm{~d} x .
\end{aligned}
$$

By integration by parts, we get

$$
\begin{aligned}
f_{n}(n z) & =\int_{0}^{1} e^{\left(x-\frac{1}{2 n}\right) n z}\left(1-x+x e^{-z}\right)^{n-1} \mathrm{~d} x \\
& =\left.\frac{\left(1-x+x e^{-z}\right)^{n}}{n\left(-1+e^{-z}\right)} e^{\left(x-\frac{1}{2 n}\right) n z}\right|_{0} ^{1}-\int_{0}^{1} \frac{n z}{n\left(-1+e^{-z}\right)} e^{\left(x-\frac{1}{2 n}\right) n z}\left(1-x+x e^{-z}\right)^{n} \mathrm{~d} x \\
& =\frac{-z e^{-z / 2}}{-1+e^{-z}} \int_{0}^{1}\left((1-x) e^{x z}+x e^{-(1-x) z}\right)^{n} \mathrm{~d} x \\
& =\frac{z / 2}{\sinh (z / 2)} \int_{0}^{1}\left((1-x) e^{x z}+x e^{-(1-x) z}\right)^{n} \mathrm{~d} x
\end{aligned}
$$

which is the claimed result.

We next have to expand the function

$$
g(x, z):=(1-x) e^{x z}+x e^{-(1-x) z}
$$

in the integrand in powers of $z$.

Lemma 4.3. We have

$$
g(x, z)=\sum_{k \geq 0} \frac{z^{k}}{k !}\left(x^{k}(1-x)+(-1)^{k} x(1-x)^{k}\right) .
$$


Proof. This follows straightforwardly from the Maclaurin series of the exponential function.

Now, set

$$
(g(x, z))^{n}:=\sum_{j \geq 0} G_{j}(n ; x) z^{j}
$$

We gather some properties of $G_{j}(n ; x)$.

Lemma 4.4. (i) For odd $j \geq 0$, we have

$$
\int_{0}^{1} G_{j}(n ; x) \mathrm{d} x=0 .
$$

(ii) For even $j \geq 0$, we have

$$
\int_{0}^{1} G_{j}(n, x) \mathrm{d} x=p_{j}(n)
$$

where $p_{j}(n)$ is a polynomial in $n$ of degree $j / 2$ and $(\ell+1)$-st highest coefficient

$$
\frac{(j / 2) !}{(j+1) ! 2^{j / 2}} q_{\ell}^{[j]}(j)
$$

with $q_{0}^{[j]}=1$ and more generally $q_{\ell}^{[j]}(x)$ is a polynomial of degree at most $3 \ell$.

Proof. Let

$$
q_{k}(x):=x^{k}(1-x)+(-1)^{k} x(1-x)^{k}
$$

and observe that $q_{k}(x)=q_{k}(1-x)$ if $k$ is even and $q_{k}(x)=-q_{k}(1-x)$ if $k$ is odd.

Now, if $j$ is odd, use the representation

$$
G_{j}(n ; x)=\sum_{j=j_{1}+\cdots+j_{n}} \prod_{\ell=1}^{n} \frac{q_{j_{\ell}}(x)}{j_{\ell} !},
$$

where $j_{\ell}$ means that we have picked the coefficient of $\left[z^{j_{\ell}}\right]$ of $g(x, z)$ in the $\ell$-th factor of the product $(g(x, z))^{n}$ when we expand it. Note that since $j$ is odd, there is always an odd number of $j_{\ell}$ 's which are odd. Thus, $G_{j}(n ; x)=-G_{j}(n ; 1-x)$ from which the first claim follows.

If $j$ is even, replace the above representation by

$$
G_{j}(n ; x)=\sum \frac{n !}{\prod_{\substack{\ell=0 \\ \ell \neq 1}}^{\infty} j_{\ell !}} \prod_{\substack{\ell=0 \\ \ell \neq 1}}^{\infty}\left(\frac{q_{\ell}(x)}{\ell !}\right)^{j_{\ell}},
$$

where $j_{\ell}$ is now the number of times the coefficient $\left[z^{\ell}\right]$ of $g(x, z)$ was picked when expanding $(g(x, z))^{n}$. Thus, the sum is over all $\left(j_{0}, j_{2}, \ldots\right)$ with $\sum \ell j_{\ell}=j$ and $\sum j_{\ell}=n$. Note that $j_{1}$ is missing since $q_{1}(x)=0$. 
Now, observe that

$$
2\left(n-j_{0}\right)=2 \sum_{\ell \geq 2} j_{\ell} \leq \sum \ell j_{\ell}=j
$$

and consequently $j_{0} \geq n-j / 2$. Thus, the integral over $G_{j}(n ; x)$ is a polynomial in $n$ of degree at most $j / 2$. To obtain the leading coefficient, one has to take $j_{0}=n-j / 2$ and $j_{2}=j / 2$ which yields

$\frac{n !}{(n-j / 2) !(j / 2) !} \cdot \frac{1}{2^{j / 2}} \cdot \int_{0}^{1} x^{j / 2}(1-x)^{j / 2} \mathrm{~d} x=\beta(j / 2+1, j / 2+1) n^{j / 2}+$ lower order terms, where $\beta(\alpha, \beta)$ denotes the beta function. Thus

$$
\frac{\beta(j / 2+1, j / 2+1)}{(j / 2) ! 2^{j / 2}}=\frac{(j / 2) !}{(j+1) ! 2^{j / 2}}
$$

as claimed.

More generally, for the $(\ell+1)$-st highest coefficient, we have to consider all the terms in 4.2 which contain $n^{j / 2-\ell}$. Such terms are, e.g., obtained by choosing $j_{0}=n-j / 2+\ell$. We will consider this case first. Also, assume that $j_{2}=j / 2-\ell-\tilde{\ell}$ and thus at most $\tilde{\ell}$ of the remaining $j_{k}$ 's are positive. Now, observe that

$$
2(j / 2-\ell-\tilde{\ell})+3 \tilde{\ell} \leq \sum_{k \geq 2} k j_{k}=j .
$$

From this, we see that $\tilde{\ell} \leq 2 \ell$ which in turn implies that the number of choices of $\left(j_{3}, \ldots\right)$ is bounded in $\ell$. Also, for each such a choice, observe that the product in 4.2 contains

$$
\frac{x^{j / 2-\ell-\tilde{\ell}}(1-x)^{j / 2-\ell-\tilde{\ell}}}{2^{j / 2-\ell-\tilde{\ell}}}
$$

since $j_{2}=j / 2-\ell-\tilde{\ell}$ and the remaining terms are of the form

$$
\prod_{\ell=3}^{\infty}\left(\frac{q_{\ell}(x)}{\ell !}\right)^{j_{\ell}}=x^{\tilde{\ell}}(1-x)^{\tilde{\ell}} q(x)
$$

where $q(x)$ is a polynomial of degree at most $2 \ell$. (This follows since $q_{k}(x)$ divided by $x(1-x)$ is a polynomial of degree $k-2$ for $k \geq 3$.) Overall, we obtain that for $j_{0}=n-j / 2+\ell$ and $j_{2}=j / 2-\ell-\tilde{\ell}$, the sum of all the terms in 4.2 has the form

$$
\frac{x^{j / 2-\ell}(1-x)^{j / 2-\ell} q(x)}{(j / 2-\ell-\widetilde{\ell}) ! 2^{j / 2}} \cdot \frac{n !}{(n-j / 2+\ell) !},
$$

where $q(x)$ is a polynomial of degree at most $2 \ell$ (which is different from the $q(x)$ above). The contribution to $p_{j}(n)$ of this is

$$
\frac{1}{(j / 2-\ell-\widetilde{\ell}) ! 2^{j / 2}}\left(\int_{0}^{1} x^{j / 2-\ell}(1-x)^{j / 2-\ell} q(x) \mathrm{d} x\right) \cdot \frac{n !}{(n-j / 2+\ell) !} .
$$


Now, the latter integral is a linear combination of beta functions, where the beta functions are of the form

$$
\beta(j / 2-\ell+k+1, j / 2-\ell+1)=\frac{(j / 2-\ell+k) !(j / 2-\ell) !}{(j-2 \ell+k+1) !},
$$

where $0 \leq k \leq 2 \ell$. Together with the factorial in 4.3 , we get

$$
\frac{(j / 2-\ell+k) !(j / 2-\ell) !}{(j / 2-\ell-\widetilde{\ell}) !(j-2 \ell+k+1) !}=\frac{(j / 2-\ell+k) !}{(j-2 \ell+k+1) !} q(j),
$$

where $q(j)$ is a polynomial in $j$ of degree at most $\tilde{\ell} \leq 2 \ell$ (again different from the $q(x)$ 's above). Note that if $0 \leq k \leq \ell$, we have

$$
\frac{(j / 2-\ell+k) !}{(j-2 \ell+k+1) !}=\frac{(j / 2) !}{(j+1) !} \cdot \frac{(j / 2) \cdots(j / 2-\ell+k+1)}{(j+1) \cdots(j-2 \ell+k+2)}
$$

and the latter fraction is a polynomial in $j$ of degree at most $\ell$. Likewise, if $\ell<k \leq 2 \ell$, we have

$$
\frac{(j / 2-\ell+k) !}{(j-2 \ell+k+1) !}=\frac{(j / 2) !}{(j+1) !} \cdot(j / 2+1) \cdots(j / 2-\ell+k)(j+1) \cdots(j-2 \ell+k+2)
$$

and the latter product is again a polynomial in $j$ of degree at most $\ell$. By combining all this, we see that 4.3 has the form

$$
\frac{(j / 2) !}{(j+1) ! 2^{j / 2}} q(j) \cdot \frac{n !}{(n-j / 2+\ell) !},
$$

where $q(j)$ is a polynomial in $j$ of degree at most $\tilde{\ell}+\ell \leq 3 \ell$ (again this $q(x)$ differs from the ones above). Also, note that this does not depend on $\tilde{\ell}$ anymore. Thus, the integral over the sum of all terms with $j_{0}=n-j / 2+\ell$ in 4.2 also has this form.

However, choosing $j_{0}=n-j / 2+\ell$ is just one way how one obtains $n^{j / 2-\ell}$ in 4.2 . Other cases arise from $j_{0}=n-j / 2+\ell-k$ with $1 \leq k \leq \ell$. Then, by the same reasoning as above, the integral over the corresponding terms in 4.2 has the form

$$
\frac{(j / 2) !}{(j+1) ! 2^{j / 2}} q(j) \cdot \frac{n !}{(n-j / 2+\ell-k) !},
$$

where $q(j)$ is a polynomial in $j$ of degree at most $3 \ell-3 k$ (here, $q(x)$ is different for different choices of $k$ ). We get the coefficient of $n^{j / 2-\ell}$ by expanding

$$
n(n-1) \cdots(n-j / 2+\ell-k+1)
$$

and picking the $k$-th highest coefficient. This coefficient can be expressed by the $k$-th elementary symmetric polynomial evaluated at $0,-1, \ldots, j / 2-\ell+k-1$ which by Newton's identity can be expressed in terms of the power function

$$
\sum_{s=1}^{j / 2-\ell+k-1} s^{r}
$$


where $1 \leq r \leq k$.

Note that the latter is polynomial in $j$ of degree $r+1$ and a simple induction shows that the above coefficient is a polynomial in $j$ of degree at most $2 k$. Combining this with the polynomial factor of (4.4) shows that the coefficient of $n^{j / 2-\ell}$ in 4.4 is of the form

$$
\frac{(j / 2) !}{(j+1) ! 2^{j / 2}} \widetilde{q}(j)
$$

where $\widetilde{q}(j)$ is a polynomial in $j$ of degree at most $3 \ell-3 k+2 k=3 \ell-k \leq 3 \ell$. Collecting all these coefficients for $0 \leq k \leq \ell$ gives now the desired result.

Now, combining everything we proved so far gives the following theorem which improves Theorem 1.1 when $a$ is an even integer.

Theorem 4.5. For even $a \geq 0$, we have

$$
S_{\operatorname{Bin}}(n)=n^{1-a / 2} \sum_{\ell=0}^{a / 2} d_{\ell, a} n^{-\ell},
$$

where

$$
d_{\ell, a}=\frac{(a / 2) !}{(a+1) 2^{a / 2}} r_{\ell}(a)
$$

with $r_{0}(x)=1$ and more generally $r_{\ell}(x)$ is a polynomial of degree at most $3 \ell$.

Proof. By definition

$$
a !\left[z^{a}\right] f_{n}(n z)=M_{a}(n) n^{a-1}=S_{\operatorname{Bin}}(n) n^{a-1},
$$

where the last step holds since $a$ is even.

Now, from Lemma 4.4 .

$$
\int_{0}^{1}(g(x, z))^{n} \mathrm{~d} x=\sum_{j \geq 0} p_{2 j}(n) z^{2 j}
$$

and since $(z / 2) / \sinh (z / 2)$ is an even function

$$
\frac{z / 2}{\sinh (z / 2)}=\sum_{j \geq 0} c_{2 j} z^{2 j}
$$

Thus, from Lemma 3.15 ,

$$
S_{\operatorname{Bin}}(n) n^{a-1}=a ! \sum_{\ell=0}^{a / 2} c_{2 \ell} p_{a-2 \ell}(n) .
$$

Next, by Lemma 4.4, it is clear that the sum is a polynomial in $n$ of degree $a / 2$. Thus, the expression can be rearranged into the claimed form. 
What is left is to prove that the coefficients of 4.5 have the claimed form. Therefore, note that the $\ell$-th coefficient of $(4.5)$ is obtained from the $(\ell-k+1)$-st highest coefficient of $p_{a-2 k}(n)$ for all $0 \leq k \leq \ell$ which by Lemma 4.4 is of the form

$$
\frac{(a / 2-k) !}{(a-2 k+1) ! 2^{a / 2-k}} q_{\ell-k}^{[a-2 k]}(a-2 k),
$$

where $q_{\ell-k}(x)$ is of degree at most $3 \ell-3 k$. Note that this coefficient has to be multiplied with $c_{2 k}$ (which is independent of $a$ ) and $a$ !. Also, note that

$$
a ! \frac{(a / 2-k) !}{(a-2 k+1) !}=\frac{(a / 2) !}{a+1} 2^{k} \prod_{i=0}^{k-1}(a-(2 i-1))
$$

and that the latter product is a polynomial in $a$ of degree $k$. Thus, (4.6) multiplied by $a$ ! and $c_{2 k}$ has the desired form and summing up all these terms gives again a term which has the desired form. This concludes the proof.

Remark 4.6. By computing $S_{\text {Bin }}(n)$ for small values of $a$ (which can either be done directly from the definition or by the recursive procedure introduced below), one can find the polynomials in the coefficients of 4.5, e.g.,

$$
r_{1}(a)=-\frac{1}{36} a^{2}-\frac{1}{6} a-\frac{1}{18}, \quad a \geq 2
$$

and

$$
r_{2}(a)=\frac{1}{2592} a^{4}+\frac{13}{3240} a^{3}+\frac{1}{810} a^{2}-\frac{29}{1620} a-\frac{13}{1080}, \quad a \geq 4 .
$$

Remark 4.7. Computing more values of $r_{\ell}(a)$ seems to suggest that the degree of these polynomials is actually $2 \ell$ and thus our bound in the previous lemma does not seem to be sharp.

Note that the proof gives the alternative representation:

$$
S_{\operatorname{Bin}}(n)=a ! n^{1-a} \sum_{\ell=0}^{a / 2} c_{2 \ell} p_{a-2 \ell}(n) .
$$

We next discuss how to compute the polynomials in this expansion in an efficient way. For this, it suffices to show that $G_{m}(n ; x)$ can be computed in a efficient way.

Proposition 4.8. We have that $G_{0}(n ; x)=1$ and for $m \geq 1$,

$$
G_{m}(n ; x)=\frac{1}{m} \sum_{\ell=2}^{m}(n \ell-m+\ell) g_{\ell}(x) G_{m-\ell}(n ; x),
$$

where

$$
g_{\ell}(x)=\frac{1}{\ell !}\left(x^{\ell}(1-x)+(-1)^{\ell} x(1-x)^{\ell}\right) .
$$


Proof. Differentiating 4.1 yields

$$
n g(x, z)^{n-1} g_{z}(x, z)=\sum_{j \geq 1} j G_{j}(x) z^{j-1}
$$

Thus,

$$
n g(x, z)^{n} z g_{z}(x, z)=g(x, z) \sum_{j \geq 0} j G_{j}(x) z^{j}
$$

Note that

$$
g(x, z)=\sum_{\ell \geq 0} g_{\ell}(x) z^{\ell} \quad \text { and } \quad z g_{z}(x, z)=\sum_{\ell \geq 0} \ell g_{\ell}(x) z^{\ell}
$$

Consequently, by reading off the coefficient of $\left[z^{m}\right]$ on both sides of (4.7):

$$
n \sum_{\ell=0}^{m} \ell g_{\ell}(x) G_{m-\ell}(x)=\sum_{\ell=0}^{m}(m-\ell) g_{\ell}(x) G_{m-\ell}(x), \quad m \geq 0 .
$$

Note that $g_{0}(x)=1$ and thus for $m \geq 1$, we can solve for $G_{m}(x)$ on the right-hand side which gives

$$
m G_{m}(x)=\sum_{\ell=1}^{m}(n \ell-m+\ell) g_{\ell}(x) G_{m-\ell}(x) .
$$

Dividing by $m$ and using that $g_{1}(x)=0$ yields the claimed result.

Moreover, the sequence of the $c_{2 j}$ 's can be actually made explicit.

Lemma 4.9. We have

$$
c_{2 j}=\frac{\left(1-2^{2 j-1}\right) B_{2 j}}{(2 j) ! 2^{2 j-1}},
$$

where $B_{2 j}$ denotes the $2 j$-th Bernoulli number.

Proof. From the well-known series expansion of $\operatorname{csch}(z)$

$$
\operatorname{csch}(z)=\frac{1}{z}-\sum_{n=1}^{\infty} \frac{2\left(2^{n-1}-1\right) B_{2 n}}{(2 n) !} z^{2 n-1}
$$

we obtain that

$$
c_{2 j}=\left[z^{2 j}\right] \frac{z / 2}{\sinh (z / 2)}=\left[z^{2 j}\right]\left(1-\sum_{n=1}^{\infty} \frac{\left(2^{2 n-1}-1\right) B_{2 n}}{(2 n) ! 2^{2 n-1}} z^{2 n}\right) .
$$

From this the result follows. 


\subsection{Poisson sum for $a$ an even integer}

In this section, we will consider 1.2 .

Similar as in the subsection above, for an integer $a \geq 0$, we define

$$
\begin{aligned}
N_{a}(n) & :=\sum_{i=0}^{n-2} \int_{0}^{\infty}\left(x-s_{i+1}\right)^{a} n e^{-n x} \frac{(n x)^{i}}{i !} \mathrm{d} x \\
& =\sum_{i=0}^{n-2} \int_{0}^{\infty}\left(x-\frac{i+1}{n}\right)^{a} n e^{-n x} \frac{(n x)^{i}}{i !} \mathrm{d} x .
\end{aligned}
$$

Also, we again define an exponential generating function:

$$
f(z):=\sum_{a \geq 0} \frac{N_{a}(n)}{a !} z^{a} .
$$

Then, we have the following lemma

Lemma 4.10. We have

$$
f(n z)=\frac{d(z)^{n}-d(z)}{d(z)-1}
$$

where $d(z)$ is the exponential generating function of the number $d_{n}$ of permutations of size $n$ with no fixed points, i.e.,

$$
d(z)=\sum_{\ell \geq 0} d_{\ell} \frac{z^{\ell}}{\ell !}
$$

Proof. Note that

$$
\begin{aligned}
f(n z) & =\sum_{a \geq 0}\left(\sum_{i=0}^{n-2} \int_{0}^{\infty}\left(x-\frac{i+1}{n}\right)^{a} n e^{-n x} \frac{(n x)^{i}}{i !} \mathrm{d} x\right) \frac{(n z)^{a}}{a !} \\
& =\sum_{i=0}^{n-2} \frac{n^{i+1}}{i !} \int_{0}^{\infty}\left(\sum_{a \geq 0} \frac{(n x z-(i+1) z)^{a}}{a !}\right) e^{-n x} x^{i} \mathrm{~d} x \\
& =\sum_{i=0}^{n-2} e^{-(i+1) z} \frac{n^{i+1}}{i !} \int_{0}^{\infty} e^{-x(1-z) n} x^{i} \mathrm{~d} x .
\end{aligned}
$$

The latter integral can be solved by the change of variable $u=x(1-z) n$ which gives

$$
\int_{0}^{\infty} e^{-x(1-z) n} x^{i} \mathrm{~d} x=((1-z) n)^{-i-1} \int_{0}^{\infty} e^{-u} u^{i} \mathrm{~d} u=i !((1-z) n)^{-i-1} .
$$

Plugging this into the expression above yields

$$
f(n z)=d(z) \sum_{i=0}^{n-2} d(z)^{i}=\frac{d(z)^{n}-d(z)}{d(z)-1}
$$

which is the claimed result. 
Next, set

$$
d(z)^{n}=\sum_{j \geq 0} p_{j}(n) z^{j}
$$

Then, the coefficients $p_{j}(n)$ have the following property.

Lemma 4.11. Let $j \neq 1$. Then $p_{j}(n)$ is a polynomial in $n$ of degree $\lfloor j / 2\rfloor$ and leading coefficient

$$
\begin{cases}1 /\left(m ! 2^{m}\right) & \text { if } j=2 m \\ 2 /\left(3(m-1) ! 2^{m}\right) & \text { if } j=2 m+1 .\end{cases}
$$

More generally, the $(\ell+1)$-st highest coefficient has the form

$$
\frac{1}{\lfloor j / 2\rfloor ! 2\lfloor j / 2\rfloor} q_{\ell}^{[j]}(\lfloor j / 2\rfloor)
$$

where $q_{\ell}^{[j]}(x)$ is a polynomial of degree at most $3 \ell+j(\bmod 2)$.

Proof. The proof is similar to that of Lemma 4.4 above. In particular, we again use the representation

$$
p_{j}(n)=\sum \frac{n !}{\prod_{\substack{\ell=0 \\ \ell \neq 1}}^{\infty} j_{\ell} !} \prod_{\substack{\ell=0 \\ \ell \neq 1}}^{\infty}\left(\frac{d_{\ell}}{\ell !}\right)^{j_{\ell}}
$$

where the sum runs over all $\left(j_{0}, j_{2}, \ldots\right)$ with $\sum \ell j_{\ell}=j$ and $\sum j_{\ell}=n$. As in the proof of Lemma 4.4, we have

$$
2\left(n-j_{0}\right)=2 \sum_{\ell \geq 2} j_{\ell} \leq \sum \ell j_{\ell}=j
$$

and thus $j_{0} \geq n-j / 2$. From this, we see that $p_{j}(n)$ is indeed a polynomial of degree $\lfloor j / 2\rfloor$ and the largest power of $n$ is reached for $j=2 m$ by choosing $j_{2}=m$ and thus

$$
\frac{n !}{(n-m) ! m !}\left(\frac{d_{2}}{2 !}\right)^{m} \sim \frac{n^{m}}{m ! 2^{m}}
$$

and for $j=2 m+1$ by choosing $j_{2}=m-1$ and $j_{3}=1$ which yields

$$
\frac{n !}{(n-m) !(m-1) !}\left(\frac{d_{2}}{2}\right)^{m-1}\left(\frac{d_{3}}{3 !}\right) \sim \frac{2 n^{m}}{3(m-1) ! 2^{m}} .
$$

Finally, that the other coefficients have the claimed form follows with similar (but simpler) arguments as in the proof of Lemma 4.4 .

We can now put everything together and prove the following identity for $S_{\text {Poi }}(n)$ which improves the result of Theorem 1.2 when $a$ is an even integer. 
Theorem 4.12. For even $a>0$, we have

$$
S_{\text {Poi }}(n)=n^{1-a / 2} \sum_{\ell=0}^{a / 2} d_{\ell, a} n^{-\ell},
$$

where

$$
d_{\ell, a}=\frac{a !}{(a / 2+1) ! 2^{a / 2}} r_{\ell}(a)
$$

with $r_{0}(x)=1$ and more generally $r_{\ell}(x)$ is a polynomial of degree at most $3 \ell$.

Proof. Note that

$$
a !\left[z^{a}\right] f(n z)=n^{a} N_{a}(n)=n^{a} S_{\mathrm{Poi}}(n)
$$

which holds since $a$ is even.

Now, set

$$
\frac{1}{d(z)-1}=\sum_{j=-2}^{\infty} c_{j} z^{j}
$$

Then, from (4.8), we have

$$
\left[z^{a}\right] \frac{d(z)^{n}-d(z)}{d(z)-1}=\sum_{\ell=-2}^{a} c_{\ell}\left(p_{a-\ell}(n)-\frac{d_{a-\ell}}{(a-\ell) !}\right) .
$$

Plugging this into 4.10 gives

$$
S_{\text {Poi }}(n)=a ! n^{-a} \sum_{\ell=-2}^{a} c_{\ell}\left(p_{a-\ell}(n)-\frac{d_{a-\ell}}{(a-\ell) !}\right) .
$$

Next, note that from Lemma 4.11, the sum is a polynomial in $n$ of degree $a / 2+1$. Consequently, we can re-arrange the above expression such that we obtain

$$
S_{\text {Poi }}(n)=n^{1-a / 2} \sum_{\ell=0}^{a / 2+1} d_{\ell, a} n^{-\ell} .
$$

What is left is to prove that $d_{a / 2+1}=0$ and that the other coefficients are of the claimed form. The latter follows with similar arguments as in Lemma 4.11. For the former, observer that

$$
d_{a / 2+1, a}=a ! \sum_{\ell=-1}^{a} c_{\ell}\left(p_{a-\ell}(0)-\frac{d_{a-\ell}}{(a-\ell) !}\right) .
$$

Next note that $p_{0}(0)=1$ and that $p_{j}(0)=0$ for $j \geq 1$ from which it follows that

$$
d_{a / 2+1, a}=-a ! \sum_{\ell=-2}^{a-1} c_{\ell} \frac{d_{a-\ell}}{(a-\ell) !}=-a !\left[z^{a}\right] \frac{d(z)-1}{d(z)-1}=0 .
$$

This concludes the proof. 
Remark 4.13. By computing $S_{\text {Poi }}(n)$ for small values of $a$ (either from the definition or the recursive method below), one can find the polynomials in 4.9 for small values of $\ell$, e.g.,

$$
r_{1}(a)=\frac{1}{18} a^{3}+\frac{1}{36} a^{2}-\frac{17}{36} a-\frac{11}{18}, \quad a \geq 2
$$

and

$$
r_{2}(a)=\frac{1}{1944} a^{6}+\frac{1}{1944} a^{5}-\frac{271}{12960} a^{4}-\frac{403}{19440} a^{3}+\frac{1499}{19440} a^{2}+\frac{253}{3240} a, \quad a \geq 4 .
$$

Remark 4.14. In contrast to the binomial sum above, now our bound for the degree of $r_{\ell}(x)$ is sharp.

The proof gives the alternative representation

$$
S_{\text {Poi }}(n)=a ! n^{-a} \sum_{\ell=-2}^{a} c_{\ell}\left(p_{a-\ell}(n)-\frac{d_{a-\ell}}{(a-\ell) !}\right) .
$$

We conclude by explaining a recursive way to compute $p_{m}(n)$.

Proposition 4.15. We have $p_{0}(n)=1$ and for $m \geq 1$,

$$
p_{m}(n)=\frac{1}{m} \sum_{\ell=2}^{m}(n \ell-m+\ell) \frac{d_{\ell}}{\ell !} p_{m-\ell}(n) .
$$

Proof. Observe that

$$
z\left(d(z)^{n}\right)^{\prime}=\sum_{j \geq 0} j p_{j}(n) z^{j}
$$

Moreover,

$$
\left(d(z)^{n}\right)^{\prime}=n d(z)^{n-1} d^{\prime}(z)
$$

and thus

$$
n d(z)^{n} z d^{\prime}(z)=d(z) \sum_{j \geq 0} j p_{j}(n) z^{j} .
$$

Reading off the $m$-th coefficient on both sides yields

$$
n \sum_{\ell=1}^{m} \frac{d_{\ell}}{(\ell-1) !} p_{m-\ell}(n)=\sum_{\ell=0}^{m} \frac{d_{\ell}}{\ell !}(m-\ell) p_{m-\ell}(n) .
$$

Rearranging gives

$$
m p_{m}(n)=\sum_{\ell=1}^{m}(n \ell-m+\ell) \frac{d_{\ell}}{\ell !} p_{m-\ell}
$$

from which the result follows. 


\section{Concluding remarks}

In this paper, we re-visited the problem of finding asymptotic expansions of (1.1) and 1.2 . First-order asymptotics were derived in several recent papers; see [3 6]. We proposed a different approach which avoids the two-stage approach from the previous studies that combined combinatorial and probabilistic tools. In particular, we showed that no case distinction is necessary between $a>0$ being an integer and $a>0$ being an arbitrary real number in order to derive the first-order asymptotics.

Our proposed approach is based on the classical Laplace method (which is the realanalytic version of the more general saddle point method; see Chapter 8 in [2]). The application of this method in the current context is slightly complicated by the fact that there is an additional integration and thus approximations have to be uniform (see Sections 2 and 3 for details). The method is able to produce further terms in the asymptotic expansion by considering more terms in the respective local limit theorems for the binomial distribution (Proposition 3.1) and Poisson distribution (Proposition 3.9). However, since for higher order terms and small values of $a$, we encounter divergent integrals (e.g., similar to the beta-integral from the proof of Lemma 3.3), we have to choose larger and larger values of $a$ when more and more terms in the asymptotic expansions are sought.

In the special case of $a>0$ being an even integer, we obtained full asymptotic expansions which turned out to be finite and moreover hold as identities. This case was also treated separately in previous works (which also implicitly contain the identities, even though the final results were formulated as first-order asymptotic results). The strategy in these previous papers was to expand the integrand and suitably rearrange terms. In this paper, we showed that the usage of exponential generating functions allows a somehow "cleaner" derivation. Moreover, with our approach, we also obtained a structural result for all coefficients in these identities (which can be used for computational purpose) and recursive means to compute the coefficients in a systematic way.

We conclude the paper by briefly commenting on the higher-dimensional case which was also studied in the literature; see e.g., 3,7]. We focus on the case where the $n$ sensors are placed uniformly at random in the $d$-dimensional unit cube and each sensor can cover a range of at most $1 /\left(n^{1 / d}\right)$ in each direction along all dimensions. (This generalizes the binomial case to higher dimensions.) Moreover, for the sake of simplicity, we assume that $n$ is a $d$-th power.

The algorithm proposed in 7] for moving the sensors to the anchor positions works recursively: first a dimension is chosen and the unit cube is divided along this dimension into $n^{1 / d}$ sub-cubes of the same size. Then, groups of $n^{(d-1) / d}$ sensors are moved to the $(d-1)$-dimensional subspaces which divide the sub-cubes along the fixed dimension into two equal parts. Finally, the same method is recursively applied to the $(d-1)$-dimensional 
subspaces. Repeating this procedure, one eventually ends up with $n^{(d-1) / d}$ tasks of moving $n^{1 / d}$ sensors on the line for which by Theorem 1.1 the complexity is asymptotic to

$$
C_{a}(n):=\frac{\Gamma(a / 2+1)}{2^{a / 2}(a+1)} n^{(d-1) / d} n^{(1-a / 2) / d}=\frac{\Gamma(a / 2+1)}{2^{a / 2}(a+1)} n^{1-a /(2 d)} .
$$

In fact, the arguments from 7 show that that the overall complexity is dominated by these 1-dimensional problems, i.e., the above term is the first term in the asymptotics of the displacement cost (the result in [7], however, was just formulated as an $\mathcal{O}$-result).

For instance, for $d=2$ and $a=2$ and $a=4$, the above expression becomes

$$
C_{2}(n)=\frac{\sqrt{n}}{6} \text { and } C_{4}(n)=\frac{1}{10} .
$$

This coincides with what was obtained in Section 3 of [7], where the above results where derived by directly evaluating the (exact) expressions for the displacement cost with mathematical software.

\section{Acknowledgments}

We thank Hsien-Kuei Hwang for bringing the topic of the current paper to our attention.

\section{References}

[1] C. Canonne, A short note on Poisson tail bounds, Retrieved from the website: http://www.cs.columbia.edu/ ccanonne/.

[2] P. Flajolet and R. Sedgewick, Analytic Combinatorics, Cambridge University Press, Cambridge, 2009.

[3] R. Kapelko, On the energy efficient displacement of random sensors for interference and connectivity, arXiv:1611.06329.

[4] _ Analysis of the threshold for energy consumption in displacement of random sensors, arXiv:1808.00794.

[5] _ Asymptotic formula for sum of moment mean deviation for order statistics from uniform distribution, Discrete Math. Algorithms Appl. 11 (2019), no. 2, $1950015,23 \mathrm{pp}$.

[6] R. Kapelko and E. Kranakis, On the displacement for covering a unit interval with randomly placed sensors, Inform. Process. Lett. 116 (2016), no. 11, 710-717.

[7] _ On the displacement for covering a d-dimensional cube with randomly placed sensors, Ad Hoc Networks 40 (2016), 37-45. 
[8] E. Kranakis, On the event distance of poisson processes with applications to sensors, Discrete Appl. Math. 179 (2014), 152-162.

[9] E. Kranakis, D. Krizanc, O. Morales-Ponce, L. Narayanan, J. Opatrny and S. Shende, Expected sum and maximum of displacement of random sensors for coverage of a domain, Proceedings of the 25th annual ACM symposium on Parallelism in algorithms and architectures, (2013), 73-82.

[10] E. Kranakis and G. Shaikhet, Displacing random sensors to avoid interference, in: Computing and Combinatorics, 501-512, Lecture Notes in Comput. Sci., 8591, Springer, Cham, 2014.

[11] _ Sensor allocation problems on the real line, J. Appl. Probab. 53 (2016), no. 3, $667-687$.

[12] M. Mitzenmacher and E. Upfal, Probability and Computing: Randomization and probabilistic techniques in algorithms and data analysis, Second edition, Cambridge Universy Press, Cambridge, 2017.

Michael Fuchs

Department of Mathematical Sciences, National Chengchi University, Taipei 116, Taiwan E-mail address: mfuchs@nccu.edu.tw

Louis Kao and Wan-Zhen $\mathrm{Wu}$

Department of Applied Mathematics, National Chiao Tung University, Hsinchu 300, Taiwan

E-mail address: chihpengkao.am03g@g2.nctu.edu.tw, 10204141@gm.nttu.edu.tw 